\title{
Adaptive Neuro-Fuzzy System to Determine the Blood Glucose Level of Diabetic
}

\author{
Auwal Nata'ala ${ }^{1}$, Hamman Dikko Muazu ${ }^{2}$, Ibrahim Goni ${ }^{3}$, Abdullahi Mohammed Jingi ${ }^{3}$ \\ ${ }^{1}$ Department of Computer Science, Faculty of Science Federal Polytechnic, Kaura-Namoda, Nigeria \\ ${ }^{2}$ Department of Operation Research, Faculty of Pure and Applied Science, Modibbo Adama University of Technology, Yola, Nigeria \\ ${ }^{3}$ Department of Computer Science, Faculty of Science Adamawa State University, Mubi, Nigeria
}

Email address:

algonis1414@gmail.com (I. Goni)

\section{To cite this article:}

Auwal Nata'ala, Hamman Dikko Muazu, Ibrahim Goni, Abdullahi Mohammed Jingi. Adaptive Neuro-Fuzzy System to Determine the Blood Glucose Level of Diabetic. Mathematics and Computer Science. Vol. 4, No. 3, 2019, pp. 63-67. doi: 10.11648/j.mcs.20190403.11

Received: March 9, 2019; Accepted: April 22, 2019; Published: October 12, 2019

\begin{abstract}
Diabetes is a chronic disease that occurs when the pancreas does not produce enough insulin. The main aim of this research work was to determine the blood glucose level of diabetic patient using adaptive Neuro-fuzzy. Data of 80 diabetic patients were collected from Federal Medical Centre Jalingo. It was used for training and testing the system, Gaussian Membership function was used, hybrid training algorithm was used for training and testing, the error obtain is 0.0008333 at epoch 4 which shows that the training performance is exactly $99.99 \%$ and testing performance of the system are $99.99 \%$ at epoch 4. This shows that adaptive Neuro-fuzzy system can be applied to medical diagnosis because of the error obtained.
\end{abstract}

Keywords: Diabetes, Neuro-Fuzzy, Gaussian, Hybrid

\section{Introduction}

Diabetes mellitus is a metabolic diseases characterized by hyperglycemia resulting from defects in insulin secretion, insulin action, or both. The chronic hyperglycemia of diabetes is associated with long-term damage, dysfunction, and failure of some different organs in human, such as the eyes, kidneys, nerves, heart, and blood vessels. The major symptoms of diabetes are Polyphagia, Blurred vision, Polyuria, Polydipsia, Weight loss, Tiredness, High Blood Glucose, Very High Blood Glucose [1]. Basically there are two types of diabetes type I and type II. Type I is medically characterized by lack of insulin while type II is medically characterized by ineffective to utilize the insulin produce by the body [2].

Computational intelligence, Artificial intelligence and soft computing techniques which includes; Artificial Neural network, fuzzy logic, evolutionary algorithms and swarm intelligence, or any combination of these that is Neuro-fuzzy, Neuro-genetics among others are widely applied to medical diagnosis, prognosis, determining severity level, controlling and predicting of any disease in recent time. Medical diagnosis largely depends on experience of the medical expert together with the results from medical laboratory scientist and the symptoms exhibit by the patient before concluding or confirming the presence of the disease. However computational intelligence/soft computing techniques follow similar procedure as medical expertise thus; the symptoms exhibit by the patient, the results collected from lab and the experience of the medical expert are assign with weights or translate into a numeric values and formulate a matrix which would be used as an inputs parameters to confirm whether or not the presence of the disease.

Soft computing techniques was used to detect diabetes in it early stage [3], Artificial Neural network applied to diabetes control was implemented in [4], Fuzzy logic was used to diagnose diabetes in [5], Neural Network and fuzzy logic and Gaussian Kernel Methodologies was used in prognosis of diabetes in [6], Adaptive Neuro-fuzzy technique was used to predict type II diabetes in [7].

In recent years many disease are diagnosis using computational intelligence, Artificial intelligence and soft computing techniques, As in the research of [8] TB was detect using Artificial Immune recognition system, [9] TB detection using fuzzy based decision support system, [10] risk assessment of cardiovascular diseases using web based 
fuzzy system, [11] combine Neural and Fuzzy logic to diagnoses hypertension, [12] diagnose malaria using ANFIS, [13] proposed neuro-fuzzy technique with online mode to medical diagnosis, [14] applied soft computing in predicting $\mathrm{TB}$, [15] combine clustering and fuzzy ruled based to medical diagnosis from dental x-ray, [16] Presented Neurofuzzy model to predict the presence of mycobacterium TB, [17] applied ANFIS to determine the severity level of osteomyelitis [18] used Neuro-fuzzy technique in TB diagnosis.

\section{Method of Data Collection}

The primary data will be collected using interview to get in-

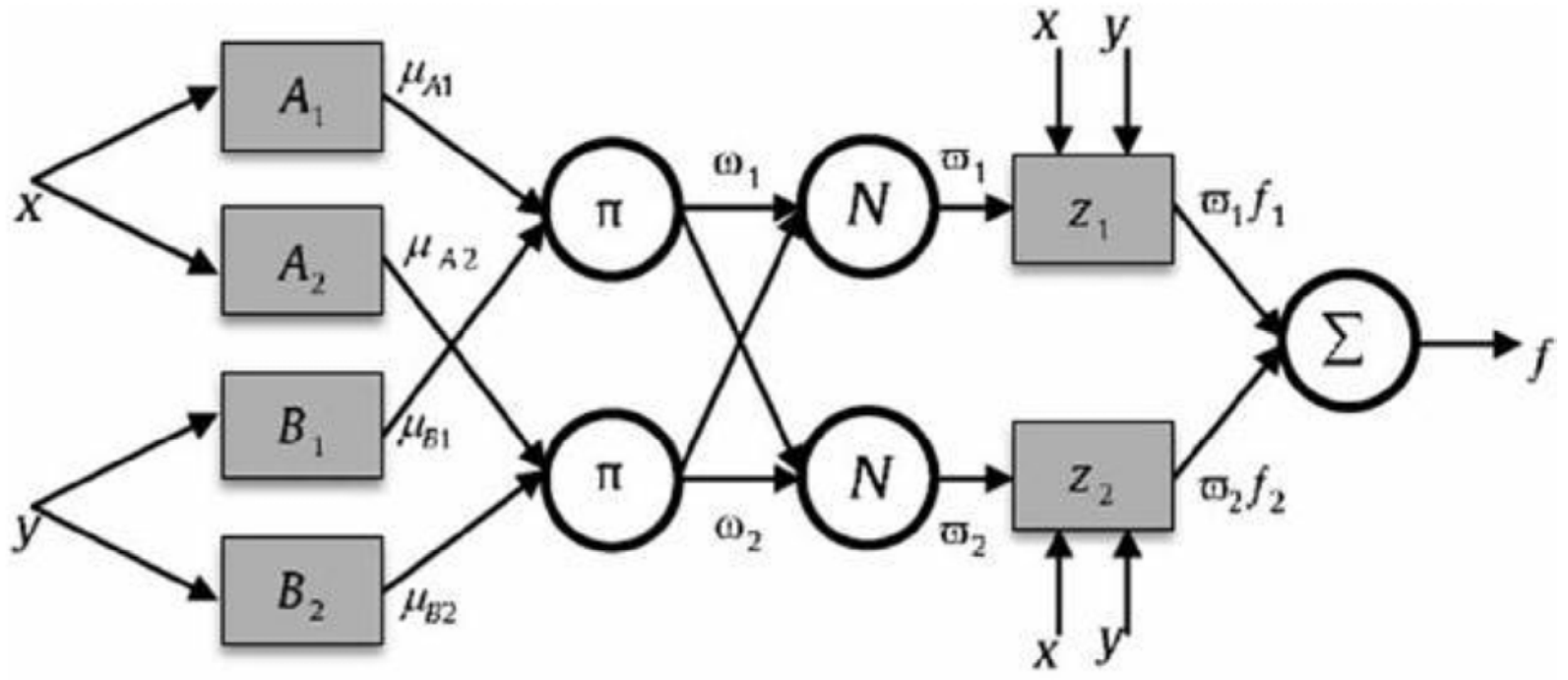

Layer 1 Layer 2 Layer 3 Layer $4 \quad$ Layer 5

Figure 1. Adaptive Neuro-fuzzy system [20].

At the first layer is the fuzzification every node has membership function $\mathrm{Ai}$ and $\mathrm{Bi}$ and the output of the layer is given as;

$$
01 i=\mu A i(x) \text { for } i=1,2
$$

The second layer is called the product layer. Each neuron in this layer is non-adaptive and computes the product of incoming signals. Each node generates the firing strength of a rule.

$$
w i=\mu(x) i \times \mu(x) i
$$

The third layer is called the normalization layer. The nodes in this layer are non-adaptive and compute the normalized firing strength which is ratio of firing strength of a rule to the sum of the firing strength of all rules.

$$
\mathrm{W}_{\mathrm{i}}=\frac{w i}{\sum w i}
$$

After receiving the normalized power from layer three the next step is for every $i-t h$ node to produce a function; depth knowledge about diabetes Mellitus from two Medical personnel while secondary data are collected from the laboratory technician's records about patients with Diabetes at Federal Medical Centre Jalingo.

\subsection{Architecture of the Proposed Model}

The proposed model will implement on a Takagi-Sugeno architecture which is a five layer architecture proposed by [19]. This architecture uses a rule structure with fuzzy antecedent and functional consequent part. The model will adopt the Gaussian membership function and the symptoms of diabetes will be analyzed and used as inputs parameters to the system. Figure 1 had shown architecture with it five layers 


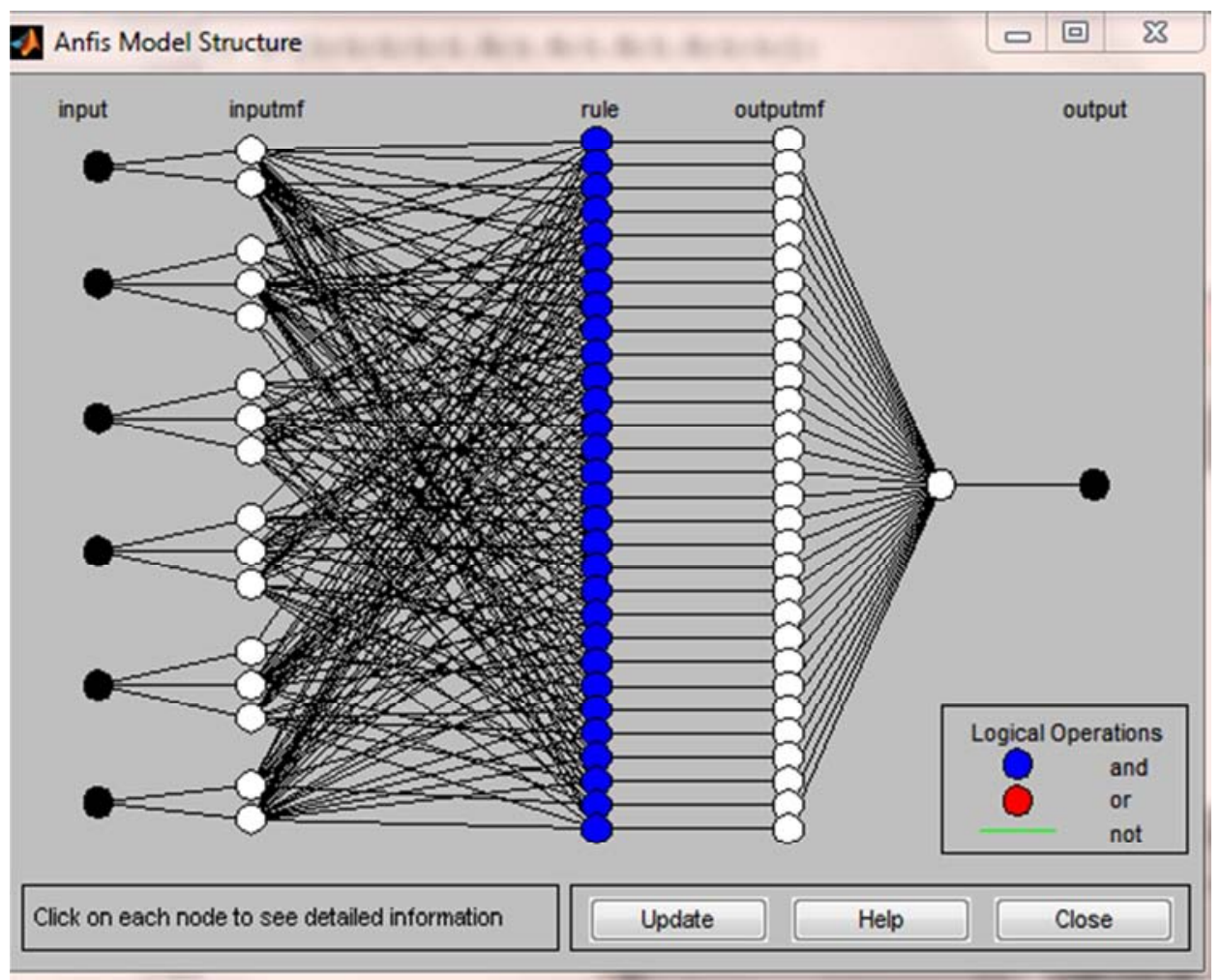

Figure 2. ANFIS for diagnosing diabetes.

The structure are obtained based on the six inputs with two linguistic terms in some inputs and three linguistic term in some were used and the rules are generated by the ANFIS and the target output is one. Figre 3 represent fuzzy logic control which is called rule viewer, the yellow color in the figure present the symptoms of the patient while the blue color indicate the diagnosis. The diagnostic output.

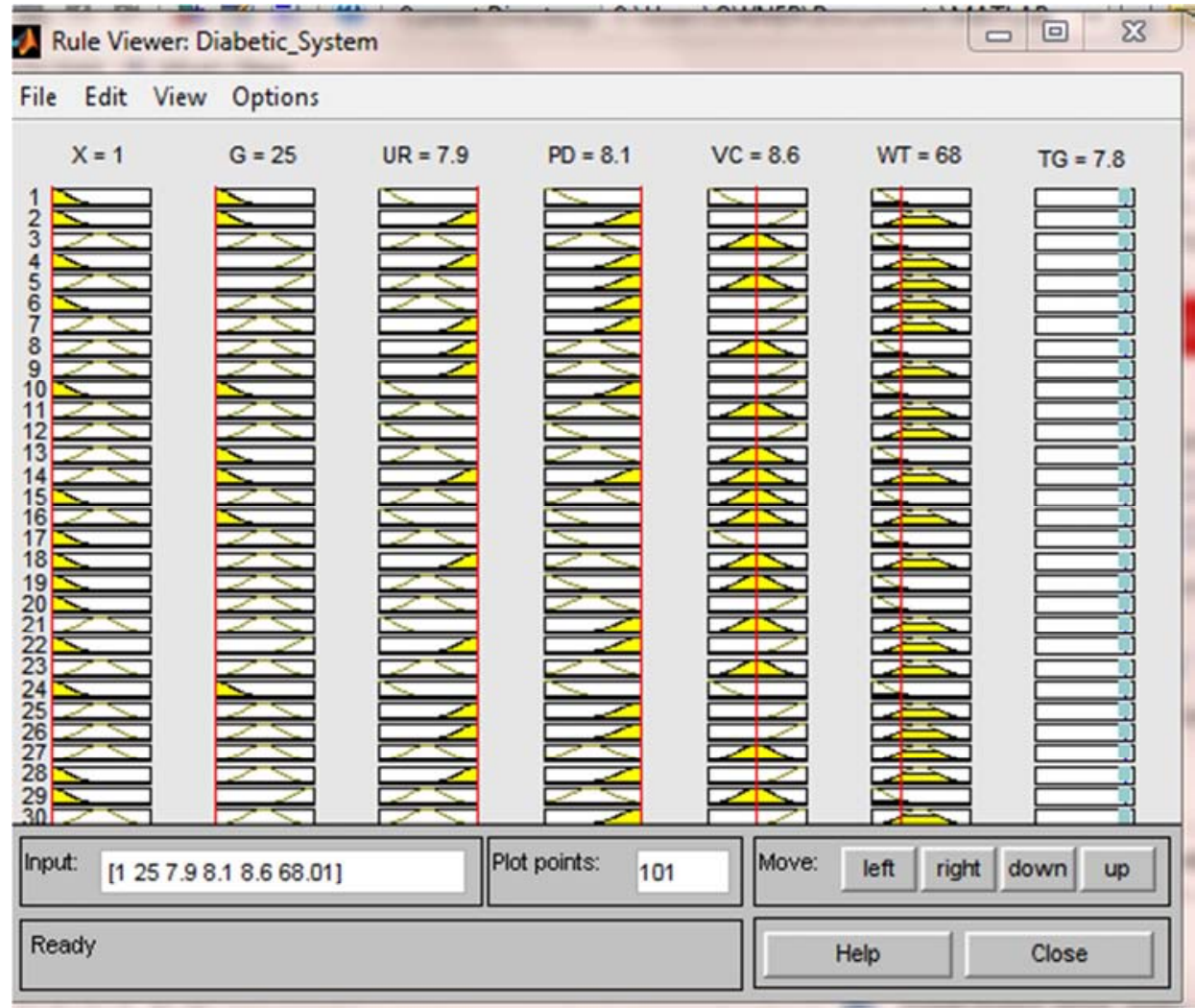

Figure 3. Rule viewer. 


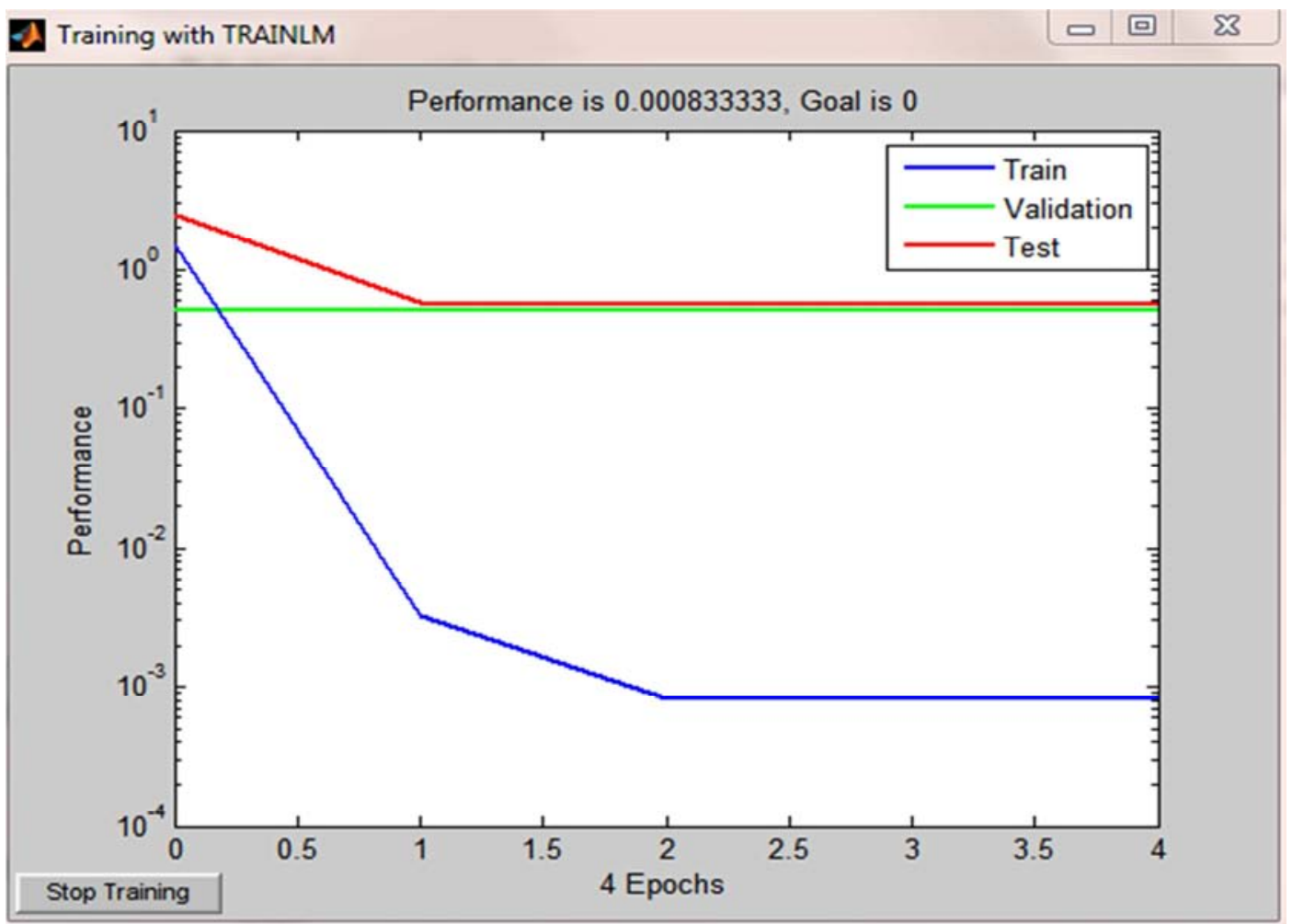

Figure 4. Validation performance.

\section{Conclusion}

In this research work ANFIS was used to implement diagnostic system for diabetic in which the 70 data was used for both the training and testing, hybrid training algorithm was used for both the training and testing of the system, ANFIS model are obtained based on these inputs, the error obtain are 0.0008333 during the training, testing and validation which shows that the training and testing performance is exactly $99.99 \%$ at epoch 4 and testing performance of the system is $99.99 \%$ at epoch 4 . The performance of this system as well as the error obtain is acceptable in medical diagnosis.

\section{Reference}

[1] American Diabetes Association (2014) Diagnosis and Classification of Diabetes Mellitus Diabetes Care Volume 37, Supplement 1, January 2014.

[2] WHO (2010) Diabetic fact sheet.

[3] Neeraj K. G., Anjali G. \& Praveen K. T. (2014). Early Detection of Diabetes Patients using Soft Computing, IEEE International Conference on Issues and Challenges in Intelligent Computing Techniques.

[4] Filipe F. \& Henrique V. (2015). Artificial Neural Networks in Diabetes Control Science and Information Conference 2015July 28-30, 2015 London, UK.

[5] Vaishali J. \& Supriya R. (2015). Improving the Prediction Rate of Diabetes using Fuzzy Expert System International
Journal of Information Technology and Computer Science, 10 (3), 84-91.

[6] Tejashri N. G. \& Satish R. T., (2015). Prognosis of Diabetes using Neural Network, Fuzzy Logic, Gaussian Kernel Method International Journal of Computer Applications, 124 (10), 3336.

[7] Alby S. \& Shivakumar BL (2018) A prediction model for type 2 diabetes using adaptive neuro-fuzzy interface system, Biomedical Research Computational Life Sciences and Smarter Technological Advancement ISSN 0970-938X.

[8] Mahmoud R. S., Shahaboddin S., Shahram G., Teh Y., Aghabozorgi S., Laiha M., \& Valentina E., (2015). RAIRS2 A new Expert System for Diagnosing Tuberculosis with Real world Tournament Selection Mechanism inside Artificial Immune recognition system, International Federation for Medical and Biological Engineering Springer, 8 (4), 11-19.

[9] Navneet W., Sharad K. T. \& Rahul M., (2015). Design and Identification of Tuberculosis using Fuzzy Based Decision Support System Advances in Computer Science and Information Technology (ACSIT), 2 (8), 57-62.

[10] Yuanda L., David P. \& Jorge M. (2015). A Web-based Fuzzy Inference System Based Tool for Cardiovascular Disease Risk Assessment NOVA. 13 (24), 7-16.

[11] Zeinab A. \& Hamid T. (2015). Design of a Fuzzy Expert System and a Multi-Layer Neural Network System For Diagnosis of Hypertension Bulletin of Environment, Pharmacology and Life Sciences, 4 (15), 138-145.

[12] Richard A., Joseph K. P. \& Kwabena R. (2015) Implementation of Adaptive Neuro Fuzzy Inference System for Malaria Diagnosis (Case Study: Kwesimintsim Polyclinic), International Journal of Computer Applications. 115 (7): 3337. 
[13] Iryna P. et al. (2016). Neo-Fuzzy Approach for Medical Diagnostics Tasks in Online-Mode, IEEE First International Conference on Data Stream Mining and Processing 23-27 August 2016, Ukraine, 34-37.

[14] Rupali Z., \& Jyoti A., (2016). Pre-Prediction of Tuberculosis Disease Using Soft Computing Technique International Journal of Advanced Research in Computer and Communication Engineering, 5 (6), 56-61.

[15] Shashank B., Praveen K. \& Amit K. G. (2016). Neuro-fuzzy expert system in e-Health Monitoring for Disease Diagnosis, International Journal of Innovations in Engineering and Technology 7 (2): 249-252.

[16] Ibrahim G., Jerome M. G. \& Timothy U. M. (2017). Designing a Neuro-Fuzzy Model for Predicting the Existence of Mycobacterium Tuberculosis $36^{\text {th }}$ Annual Conference of the Nigerian Mathematical Society (NMS) $9^{\text {th }}-12^{\text {th }}$ May 2017 held at University of Agriculture Makurdi, Benue State.
[17] Jerome M. G, Ibrahim Goni \& Timothy U. M. (2017) Adaptive Neuro-fuzzy system for Determining the Severity Level of Osteomyelitis and Control, Archives of Applied Science Research, 9 (2), 9-15.

[18] Jerome M. G., Ibrahim G. \& Mohammed I. (2018). NeuroFuzzy Approach for Diagnosing and Control of Tuberculosis the International Journal of Computational Science, Information Technology and Control Engineering (IJCSITCE) $5(1), 1-10$.

[19] Jang, J. S. R., Sun, \& Mizutani, E. (1997). Neuro-Fuzzy and Soft Computing: A Computational Approach to Learning and Machine Intelligence. NJ Prentice-Hall Ltd USA.

[20] Michael N. (2005). Artificial Intelligence A Guide to Intelligent Systems $2^{\text {nd }}$ Edition Addison Wisely Pearson Education Limited England. 